IFT-P.072/2000

\title{
Covariant Quantization of the Superstring
}

\author{
Nathan Berkovits 1 \\ Instituto de Física Teórica, Universidade Estadual Paulista \\ Rua Pamplona 145, 01405-900, São Paulo, SP, Brasil
}

\begin{abstract}
After reviewing the Green-Schwarz superstring using the approach of Siegel, the superstring is covariantly quantized by constructing a BRST operator from the fermionic constraints and a bosonic pure spinor ghost variable. Physical massless vertex operators are constructed and, for the first time, N-point tree amplitudes are computed in a manifestly ten-dimensional super-Poincaré covariant manner. Quantization can be generalized to curved supergravity backgrounds and the vertex operator for fluctuations around $A d S_{5} \times S^{5}$ is explicitly constructed. This review is written in a self-contained manner and is based on talks given at the Fradkin Memorial Conference and Strings 2000.
\end{abstract}

August 2000

1 e-mail: nberkovi@ift.unesp.br 


\section{Introduction}

There are many motivations for covariantly quantizing the superstring. As in any theory, it is desirable to make all physical symmetries manifest in order to reduce the amount of calculations and simplify any cancellations coming from the symmetry. A second motivation comes from the desire to construct a quantizable sigma model action for the superstring in curved backgrounds with Ramond-Ramond flux. A third motivation is that a more symmetrical formulation of superstring theory may shed light on some of the mysteries of M-theory.

Although the light-cone Green-Schwarz (GS) formalism [1] has been useful for computing four-point tree-level and one-loop amplitudes, it is impractical for computing higherpoint or higher-loop amplitudes because of dependence on the locations of the light-cone interaction points. Using the covariant RNS formalism, one can easily compute N-point tree-level and one-loop amplitudes involving bosons, but amplitudes involving fermions are complicated by the presence of spin fields [2]. Furthermore, lack of manifest spacetime supersymmetry in the RNS expressions complicates the analysis of finiteness properties and, for more than one-loop, leads to picture-changing problems.

For more than 15 years, a classical super-Poincaré covariant version of the GS formalism [3] has existed. But until the recent work described here, quantization problems have prevented this formalism from being used to compute non-vanishing scattering amplitudes. In this review, the covariant GS formalism is quantized by constructing a BRST operator from the fermionic constraints and a bosonic pure spinor ghost variable. After constructing physical massless vertex operators, N-point tree amplitudes are computed for the first time in a manifestly ten-dimensional super-Poincaré covariant manner.

In previous papers, this author has discussed quantization of the superstring compactified to four [ [ब or six [5] dimensions using "hybrid" variables which combine four or six-dimensional GS variables with a $c=9$ or $c=6$ superconformal field theory for the compactification. These hybrid formalisms have manifest four or six-dimensional superPoincaré covariance, $\mathrm{N}=2$ worldsheet supersymmetry, and are related to the usual RNS formalism by a field redefinition. Unfortunately, the precise relation between these hybrid formalisms and the new ten-dimensional super-Poincaré covariant formalism is still unclear, so this review will not discuss the hybrid formalisms.

In section 2 of this paper, the covariant GS formalism will be reviewed using the approach of Siegel [6] where the canonical momentum to $\theta^{\alpha}$ is an independent variable. 
In section 3 , it will be argued [7] that a bosonic pure spinor variable, $\lambda^{\alpha}$, plays the role of the worldsheet ghost in this formalism, and a BRST operator is constructed out of the fermionic constraints and $\lambda^{\alpha}$. In section 4 , physical massless vertex operators will be constructed and, in section 5 , they will be used to compute N-point tree amplitudes [7] [8]. These amplitudes are manifestly super-Poincaré covariant and involve integration over an on-shell 'harmonic' superspace including five $\theta$ 's and three $\lambda$ 's. In section 6 , the cohomology of the BRST operator is shown to reproduce the light-cone GS spectrum [9]. In section 7 , this quantization method is generalized to curved supergravity backgrounds [7] and the vertex operator is constructed for fluctuations around an $A d S_{5} \times S^{5}$ background with Ramond-Ramond flux [10]. In section 8, some open problems and applications are discussed.

\section{Review of GS Formalism using the Approach of Siegel}

In conformal gauge, the classical covariant GS action for the heterotic superstring is [3]

$$
S=\int d^{2} z\left[\frac{1}{2} \Pi^{m} \bar{\Pi}_{m}+\frac{1}{4} \Pi_{m} \theta^{\alpha} \gamma_{\alpha \beta}^{m} \bar{\partial} \theta^{\beta}-\frac{1}{4} \bar{\Pi}_{m} \theta^{\alpha} \gamma_{\alpha \beta}^{m} \partial \theta^{\beta}\right]+S_{R}
$$

where $x^{m}$ and $\theta^{\alpha}$ are the $d=10$ worldsheet variables ( $m=0$ to $9, \alpha=1$ to 16 ), $S_{R}$ describes the right-moving degrees of freedom for the $E_{8} \times E_{8}$ or $S O(32)$ lattice, and $\Pi^{m}=\partial x^{m}+\frac{1}{2} \theta^{\alpha} \gamma_{\alpha \beta}^{m} \partial \theta^{\beta}$ and $\bar{\Pi}^{m}=\bar{\partial} x^{m}+\frac{1}{2} \theta^{\alpha} \gamma_{\alpha \beta}^{m} \bar{\partial} \theta^{\beta}$ are supersymmetric combinations of the momentum. Note that $\gamma_{\alpha \beta}^{m}$ and $\gamma^{m \alpha \beta}$ are $16 \times 16$ symmetric matrices satisfying $\gamma_{\alpha \beta}^{(m} \gamma^{n) \beta \gamma}=2 \eta^{m n} \delta_{\alpha}^{\gamma}$ and are the off-diagonal blocks in the Weyl representation of the $32 \times 32$ ten-dimensional $\Gamma^{m}$-matrices. In what follows, the right-moving degrees of freedom play no role and will be ignored. Also, all of the following remarks are easily generalized to the Type I and Type II superstrings.

Since the action of (2.1) is in conformal gauge, it needs to be supplemented with the Virasoro constraint $T=-\frac{1}{2} \Pi^{m} \Pi_{m}=0$. Also, since the canonical momentum to $\theta^{\alpha}$ does not appear in the action, one has the Dirac constraint $p_{\alpha}=\delta \mathrm{E} / \delta \partial_{0} \theta^{\alpha}=\frac{1}{2}\left(\Pi_{m}-\right.$ $\left.\frac{1}{4} \theta \gamma_{m} \partial_{1} \theta\right)\left(\gamma^{m} \theta\right)_{\alpha}$ where $p_{\alpha}$ is the canonical momentum to $\theta^{\alpha}$. If one defines

$$
d_{\alpha}=p_{\alpha}-\frac{1}{2}\left(\Pi_{m}-\frac{1}{4} \theta \gamma_{m} \partial_{1} \theta\right)\left(\gamma^{m} \theta\right)_{\alpha}
$$

one can use the canonical commutation relations to find $\left\{d_{\alpha}, d_{\beta}\right\}=-\gamma_{\alpha \beta}^{m} \Pi_{m}$, which implies (since $\Pi^{m} \Pi_{m}=0$ is a constraint) that the sixteen Dirac constraints $d_{\alpha}$ have eight first-class 
components and eight second-class components. Since the anti-commutator of the secondclass constraints is non-trivial (i.e. the anti-commutator is an operator $\Pi^{+}$rather than a constant), standard Dirac quantization cannot be used since it would involve inverting an operator. So except in light-cone gauge (where the commutator becomes a constant), the covariant Green-Schwarz formalism cannot be easily quantized.

In 1986, Siegel suggested an alternative approach in which the canonical momentum to $\theta^{\alpha}$ is an independent variable using the free-field action [6]

$$
S=\int d^{2} z\left[\frac{1}{2} \partial x^{m} \bar{\partial} x_{m}+p_{\alpha} \bar{\partial} \theta^{\alpha}\right]
$$

In this approach, Siegel attempted to replace the problematic constraints of the covariant GS action with some suitable set of first-class constraints constructed out of the supersymmetric objects $\left(\Pi^{m}, d_{\alpha}, \partial \theta^{\alpha}\right)$ where

$$
d_{\alpha}=p_{\alpha}-\frac{1}{2}\left(\Pi^{m}-\frac{1}{4} \theta \gamma^{m} \partial \theta\right)\left(\gamma_{m} \theta\right)_{\alpha}
$$

is defined as in (2.2) and is no longer constrained to vanish. The first-class constraints should include the Virasoro constraint $T=-\frac{1}{2} \Pi^{m} \Pi_{m}-d_{\alpha} \partial \theta^{\alpha}=-\frac{1}{2} \partial x^{m} \partial x_{m}-p_{\alpha} \partial \theta^{\alpha}$, and the first-class part of the $d_{\alpha}$ constraint, which is the irreducible part of $G^{\alpha}=\Pi^{m}\left(\gamma_{m} d\right)^{\alpha}$. To get to light-cone gauge, one also needs constraints such as $C^{m n p}=d_{\alpha}\left(\gamma^{m n p}\right)^{\alpha \beta} d_{\beta}$ which is supposed to replace the second-class constraints in $d_{\alpha}$. Although this approach was successfully used for quantizing the superparticle [11], a set of constraints which closes at the quantum level and which reproduces the correct physical superstring spectrum was never found.

Nevertheless, the approach of Siegel has the advantage that all worldsheet fields are free which makes it trivial to compute the OPE's that

$$
x^{m}(y) x^{n}(z) \rightarrow-2 \eta^{m n} \log |y-z|, \quad p_{\alpha}(y) \theta^{\beta}(z) \rightarrow \delta_{\alpha}^{\beta}(y-z)^{-1} .
$$

This gives some useful clues about the appropriate ghost degrees of freedom. Since $\left(\theta^{\alpha}, p_{\alpha}\right)$ contributes -32 to the conformal anomaly, the total matter contribution is -22 which is expected to be cancelled by a ghost contribution of +22 . Furthermore, the spin contribution to the $S O(9,1)$ Lorentz currents in Siegel's approach is $M_{m n}=\frac{1}{2} p \gamma_{m n} \theta$, as compared with the spin contribution to the $S O(9,1)$ Lorentz currents in the RNS formalism which is $\psi_{m} \psi_{n}$. These two Lorentz currents satisfy similar OPE's except for the numerator in the double pole of $M_{m n}$ with $M_{m n}$, which is +4 in Siegel's approach and +1 in the RNS formalism. This suggests that the worldsheet ghosts should have Lorentz currents which contribute -3 to the double pole. 


\section{Quantization using Pure Spinors}

In fact, there exists an $S O(9,1)$ irreducible representation contributing $c=22$ and with a -3 coefficient in the double pole of its Lorentz current [7]. This representation is a complex bosonic spinor $\lambda^{\alpha}$ satisfying the "pure spinor" condition that

$$
\lambda^{\alpha} \gamma_{\alpha \beta}^{m} \lambda^{\beta}=0
$$

for $m=0$ to 9. Pure spinors have previously been used by Howe [12] for describing super-Yang-Mills and supergravity equations of motion as integrability conditions, and by Nilsson [13] as superspace auxiliary fields.

There are eleven independent complex degrees of freedom in $\lambda^{\alpha}$, as can be seen by temporarily breaking $S O(9,1)$ to $S O(8)$ and solving the constraint of (3.1). If $\gamma^{ \pm}=$ $\frac{1}{2}\left(\gamma^{0} \pm \gamma^{9}\right)$ and $\sigma_{a \dot{a}}^{j}$ are the $S O(8)$ Pauli matrices satisfying $\sigma_{a \dot{a}}^{(j} \sigma_{a \dot{b}}^{k)}=2 \delta^{j k} \delta_{\dot{a} \dot{b}}$ for $(j, a, \dot{a})=1$ to 8 , then $\lambda \gamma^{-} \lambda=0$ implies that $s^{a}=\left(\gamma^{+} \lambda\right)^{a}$ is a null complex $S O(8)$ spinor satisfying $s^{a} s^{a}=0$. Furthermore, $\lambda \gamma^{j} \lambda=0$ implies that $s^{a} \sigma_{a \dot{a}}^{j}\left(\gamma^{-} \lambda\right)^{\dot{a}}=0$, which implies that $\left(\gamma^{-} \lambda\right)^{\dot{a}}=v^{j}\left(\sigma^{j} s\right)^{\dot{a}}$ for some complex vector $v^{j} \cdot \lambda \gamma^{+} \lambda=0$ gives no new constraints on $s^{a}$ and $v^{j}$. But this parameterization of $\lambda^{\alpha}$ is invariant under the gauge transformation $\delta v^{j}=\epsilon^{\dot{a}}\left(\sigma^{j} s\right)^{\dot{a}}$, which allows one to gauge away half of the components of $v^{j}$. So a pure spinor $\lambda^{\alpha}$ can be parameterized by the seven complex components of a null $s^{a}$ and the four remaining complex components of $v^{j}$ [9]. After Wick rotation to $S O(10)$, one can alternatively describe $\lambda^{\alpha}$ as a complex scale parameter multiplying the complex tendimensional coset space $S O(10) / U(5)[7]$.

Using either the $S O(8)$ or $S O(10) / U(5)$ descriptions of $\lambda^{\alpha}$, a free field action $S_{\lambda}$ can be constructed out of the eleven left-moving unconstrained parameters and their canonical momenta with conformal anomaly $c=22$. Although the unconstrained parameters do not transform covariantly under $S O(9,1)$, the only combinations which appear in the vertex operators are $\lambda^{\alpha}$ and its Lorentz current $N_{m n}$, which satisfy the manifestly $\operatorname{SO}(9,1)$ covariant OPE's

$$
N_{m n}(y) \lambda^{\alpha}(z) \rightarrow \frac{\frac{1}{2}\left(\gamma^{m n} \lambda\right)^{\alpha}}{y-z}, \quad N_{k l}(y) N_{m n}(z) \rightarrow \frac{\eta_{m[l} N_{k] n}-\eta_{n[l} N_{k] m}}{y-z}-3 \frac{\eta_{k[n} \eta_{m] l}}{(y-z)^{2}}
$$

One can alternatively obtain the OPE's of (3.2) from an $\mathrm{SO}(9,1)$ WZW model of level -3 , however, $\lambda^{a}$ would not be a fundamental field in such an action [14]. 
One still needs to define which states are physical in this Hilbert space. The physical state condition will be defined as ghost-number one states in the cohomology of the BRSTlike operator [7]

$$
Q=\int d z \lambda^{\alpha}(z) d_{\alpha}(z)
$$

where $\lambda^{\alpha}$ carries ghost-number one and $d_{\alpha}$ is defined in (2.4). Since $d_{\alpha}$ satisfies the OPE $d_{\alpha}(y) d_{\beta}(z) \rightarrow-(y-z)^{-1} \gamma_{\alpha \beta}^{m} \Pi_{m}$, the pure spinor condition on $\lambda^{\alpha}$ implies that $Q$ is nilpotent. Although it is a bit unusual that $Q$ is constructed from second-class constraints, it will be shown in section 5 that its cohomology reproduces the correct light-cone GS spectrum.

\section{Physical Massless Vertex Operators}

Since there is no tachyon, the massless open superstring state is constructed from zero modes of the dimension-zero worldsheet fields $x^{m}, \theta^{\alpha}$, and $\lambda^{\alpha}$. Since it has ghost-number one, it can be written as [7]

$$
U=\lambda^{\alpha} A_{\alpha}(x, \theta)
$$

where $A_{\alpha}(x, \theta)$ is a spinor superfield. Since $d_{\alpha}(y) A_{\beta}(x, \theta) \rightarrow(y-z)^{-1} D_{\alpha} A_{\beta}(x, \theta)$ where $D_{\alpha}=\frac{\partial}{\partial \theta^{\alpha}}+\left(\gamma^{m} \theta\right)_{\alpha} \partial_{m}$ is the superspace derivative, $Q U=0$ implies that $\lambda^{\alpha} \lambda^{\beta} D_{\alpha} A_{\beta}=0$. But since $\lambda^{\alpha} \lambda^{\beta}=\frac{1}{32}\left(\gamma^{m n p q r}\right)^{\alpha \beta}\left(\lambda \gamma_{m n p q r} \lambda\right)$, this implies that $\left(\gamma_{m n p q r}\right)^{\alpha \beta} D_{\alpha} A_{\beta}=0$ for every five-form direction mnpqr. Furthermore, the gauge invariance $\delta U=Q \Omega$ implies that $A_{\alpha}$ has the gauge transformation $\delta A_{\alpha}=D_{\alpha} \Omega$.

The equation $\left(\gamma_{m n p q r}\right)^{\alpha \beta} D_{\alpha} A_{\beta}=0$ and gauge invariance $\delta A_{\alpha}=D_{\alpha} \Omega$ describe the spinor gauge superfield for linearized on-shell $d=10$ super-Yang-Mills [15]. It can be gauge-fixed to the form

$$
A_{\alpha}(x, \theta)=a_{m}(x)\left(\gamma^{m} \theta\right)_{\alpha}+\left(\xi(x) \gamma_{m n p}\right)_{\alpha}\left(\theta \gamma^{m n p} \theta\right)+\ldots
$$

where $a_{m}(x)$ and $\xi^{\alpha}(x)$ are the on-shell gluon and gluino and all the components in ... are auxiliary fields which are related to $a_{m}$ and $\xi^{\alpha}$ by equations of motion.

To compute scattering amplitudes, one also needs vertex operators in integrated form, $\int d z V$, where $V$ is usually obtained from the unintegrated vertex operator $U$ by anticommuting with the $b$ ghost. But since there is no natural candidate for the $b$ ghost in this formalism, one needs to use an alternative method for obtaining $V$ which is from the 
relation $[Q, V]=\partial U[16]$. Using this alternative method, one finds for the open superstring massless vertex operator that [7]

$$
V=A_{\alpha}(x, \theta) \partial \theta^{\alpha}+A_{m}(x, \theta) \Pi^{m}+W^{\alpha}(x, \theta) d_{\alpha}+F^{m n}(x, \theta) N_{m n},
$$

where $A_{m}=\frac{1}{8} \gamma_{m}^{\alpha \beta} D_{\alpha} A_{\beta}, W^{\alpha}=\frac{1}{10} \gamma_{m}^{\alpha \beta}\left(D_{\beta} A^{m}-\partial^{m} A_{\beta}\right)$, and $F^{m n}=\partial^{[m} A^{n]}$. It will be useful to note that in components,

$$
V=a_{m}(x) \partial x^{m}+\partial_{[m} a_{n]}(x) M^{m n}+\xi^{\alpha}(x) q_{\alpha}+O\left(\theta^{2}\right),
$$

where $M^{m n}=\frac{1}{2} p \gamma^{m n} \theta+N^{m n}$ is the spin contribution to the Lorentz current and $q_{\alpha}=p_{\alpha}+\frac{1}{2}\left(\partial x^{m}+\frac{1}{12} \theta \gamma^{m} \theta\right)\left(\gamma_{m} \theta\right)_{\alpha}$ is the spacetime-supersymmetry current, so (4.4) closely resembles the RNS vertex operator [2] for the gluon and gluino. If one drops the $F^{m n} N_{m n}$ term, the vertex operator of (4.3) was suggested by Siegel [6] based on superspace arguments.

For the closed superstring, the massless vertex operator is $U=\lambda^{\alpha} \widehat{\lambda}^{\widehat{\beta}} A_{\alpha \widehat{\beta}}(x, \theta, \widehat{\theta})$ where $\widehat{\lambda}^{\widehat{\alpha}}$ and $\widehat{\theta^{\alpha}}$ are right-moving worldsheet fields and the chirality of the $\widehat{\alpha}$ index depends if the superstring is IIA or IIB. The physical state condition $Q U=\widehat{Q} U=0$ and gauge invariance $\delta U=Q \widehat{\Omega}+\widehat{Q} \Omega$ where $\widehat{Q} \widehat{\Omega}=Q \Omega=0$ implies that 10

$$
\begin{gathered}
\gamma_{m n p q r}^{\alpha \beta} D_{\alpha} A_{\beta \widehat{\gamma}}=\gamma_{m n p q r}^{\widehat{\alpha} \gamma} \widehat{D}_{\widehat{\alpha}} A_{\beta \widehat{\gamma}}=0, \quad \delta A_{\alpha \widehat{\beta}}=D_{\alpha} \widehat{\Omega}_{\widehat{\beta}}+\widehat{D}_{\widehat{\beta}} \Omega_{\alpha} \\
\gamma_{m n p q r}^{\alpha \beta} D_{\alpha} \Omega_{\beta}=\gamma_{m n p q r}^{\widehat{\alpha} \widehat{\gamma}} \widehat{D}_{\widehat{\alpha}} \widehat{\Omega}_{\widehat{\gamma}}=0
\end{gathered}
$$

for any five-form direction mnpqr, which are the linearized equations of motion and gauge invariances of the Type IIA or Type IIB supergravity multiplet. The integrated form of the closed superstring massless vertex operator is the left-right product of the open superstring vertex operator of (4.3) and will be used in section 7 for quantizing the superstring in a curved supergravity background.

\section{Tree-Level Massless Scattering Amplitudes}

As usual, the $N$-point tree-level open superstring scattering amplitude will be defined as the correlation function of 3 unintegrated vertex operators $U_{r}$ and $N-3$ integrated vertex operators $\int d z V_{r}$. For massless external states, these vertex operators are given in (4.1) and (4.3). 
The first step to evaluate the correlation function is to eliminate all worldsheet fields of non-zero dimension (i.e. $\partial x^{m}, \partial \theta^{\alpha}, p_{\alpha}$ and $N^{m n}$ ) by using their OPE's with other worldsheet fields and the fact that they vanish at $z \rightarrow \infty$. One then integrates over the $x^{m}$ zero modes to get a Koba-Nielson type formula,

$$
A=\int d z_{4} \ldots d z_{N}\left\langle\lambda^{\alpha} \lambda^{\beta} \lambda^{\gamma} f_{\alpha \beta \gamma}\left(z_{r}, k_{r}, \eta_{r}, \theta\right)\right\rangle
$$

where $\lambda^{\alpha} \lambda^{\beta} \lambda^{\gamma}$ comes from the three unintegrated vertex operators and $f_{\alpha \beta \gamma}$ is some function of the $z_{r}$ 's, the momenta $k_{r}$, the polarizations $\eta_{r}$, and the remaining $\theta$ zero modes.

One would like to define the correlation function $\left\langle\lambda^{\alpha} \lambda^{\beta} \lambda^{\gamma} f_{\alpha \beta \gamma}\right\rangle$ such that $A$ is supersymmetric and gauge invariant. An obvious way to make $A$ supersymmetric is to require that the correlation function vanishes unless all sixteen $\theta$ zero modes are present, but this gives the wrong answer by dimensional analysis. The correct answer comes from realizing that $Y=\lambda^{\alpha} \lambda^{\beta} \lambda^{\gamma} f_{\alpha \beta \gamma}$ satisfies the constraint $Q Y=0$ since the external states are on-shell. Furthermore, gauge invariance implies that $\langle Y\rangle$ should vanish whenever $Y=Q \Omega$.

At zero momentum and ghost-number three, there is precisely one state in the cohomology of $Q$ which is $\left(\lambda \gamma^{m} \theta\right)\left(\lambda \gamma^{n} \theta\right)\left(\lambda \gamma^{p} \theta\right)\left(\theta \gamma_{m n p} \theta\right)$. So if

$$
f_{\alpha \beta \gamma}(\theta)=A_{\alpha \beta \gamma}+\theta^{\delta} B_{\alpha \beta \gamma \delta}+\ldots+\left(\gamma^{m} \theta\right)_{\alpha}\left(\gamma^{n} \theta\right)_{\beta}\left(\gamma^{p} \theta\right)_{\gamma}\left(\theta \gamma_{m n p} \theta\right) F+\ldots,
$$

it is natural to define

$$
\left\langle\lambda^{\alpha} \lambda^{\beta} \lambda^{\gamma} f_{\alpha \beta \gamma}\left(z_{r}, k_{r}, \eta_{r}, \theta\right)\right\rangle=F\left(z_{r}, k_{r}, \eta_{r}\right)
$$

This definition is supersymmetric since $\left(\lambda \gamma^{m} \theta\right)\left(\lambda \gamma^{n} \theta\right)\left(\lambda \gamma^{p} \theta\right)\left(\theta \gamma_{m n p} \theta\right) \theta^{\alpha}$ is not annihilated by $Q$, and is gauge invariant since $\left(\lambda \gamma^{m} \theta\right)\left(\lambda \gamma^{n} \theta\right)\left(\lambda \gamma^{p} \theta\right)\left(\theta \gamma_{m n p} \theta\right) \neq Q \Omega$. Note that (5.3) can be interpreted as integration over an on-shell harmonic superspace involving five $\theta$ 's since $\left\langle\lambda^{\alpha} \lambda^{\beta} \lambda^{\gamma} f_{\alpha \beta \gamma}\right\rangle=\int\left(d \theta \gamma_{m}\right)^{\alpha}\left(d \theta \gamma_{n}\right)^{\beta}\left(d \theta \gamma_{p}\right)^{\gamma}\left(d \theta \gamma^{m n p} d \theta\right) f_{\alpha \beta \gamma}[7]$.

For three-point scattering, $A=\left\langle\lambda^{a} A_{\alpha}^{1} \lambda^{\beta} A_{\beta}^{2} \lambda^{\gamma} A_{\gamma}^{3}\right\rangle$, it is easy to check that the prescription of (5.3) reproduces the usual super-Yang-Mills cubic vertex. In the gauge of (4.2), each $A_{\alpha}$ contributes one, two or three $\theta$ 's. If the five $\theta$ 's are distributed as $(1,1,3)$, one gets the $a_{m}^{1} a_{n}^{2} \partial^{[m} a^{3 n]}$ vertex, whereas if they are distributed as $(2,2,1)$, one gets the $\left(\xi^{1} \gamma^{m} \xi^{2}\right) a_{m}^{3}$ vertex. Together with Brenno Vallilo, it was proven that the above prescription agrees with the standard RNS prescription of [2] for N-point massless tree amplitudes involving up to four fermions [ [8]. The relation of (4.4) to the RNS massless vertex operator was used in this proof, and the restriction on the number of fermions comes from the need for different pictures in the RNS prescription. 


\section{Cohomology of $\mathrm{Q}$}

To compute the cohomology of $Q=\int d z \lambda^{\alpha} d_{\alpha}$, it is convenient to use the $S O(8)$ parameterization of $\lambda^{\alpha}$ in terms of $s^{a}$ and $v^{j}$ discussed in section 3. The gauge invariance $\delta v^{j}=\epsilon^{\dot{a}}\left(\sigma^{j} s\right)^{\dot{a}}$ leads to a new fermionic ghost anti-chiral spinor $t^{\dot{a}}$, and the invariance of the gauge parameter, $\delta \epsilon^{\dot{a}}=y^{j}\left(\sigma^{j} s\right)^{\dot{a}}$, leads to a new bosonic ghost-for-ghost vector $v_{(1)}^{j}$. These gauge invariances continue indefinitely until one has an infinite chain of bosonic vectors $v_{(n)}^{j}$ and fermionic anti-chiral spinors $t_{(n)}^{j}$ for $n=0$ to $\infty$ where $n=0$ labels the original vector and spinor [9].

In terms of these left-moving dimension-zero worldsheet fields, $Q=\int d z s^{a} G^{a}$ where

$$
G^{a}=\left(\gamma^{-} d\right)^{a}+\sigma_{j}^{a \dot{a}}\left[v_{(0)}^{j}\left(\gamma^{+} d\right)^{\dot{a}}+\sum_{n=0}^{\infty}\left(w_{(n)}^{j} t_{(n)}^{\dot{a}}+v_{(n+1)}^{j} u_{(n)}^{\dot{a}}\right)\right]
$$

$\left(w_{(n)}^{j}, u_{(n)}^{\dot{a}}\right)$ are the canonical momenta for $\left(v_{(n)}^{j}, t_{(n)}^{\dot{a}}\right)$, and the infinite sum comes from the gauge invariances. Note that $Q^{2}=0$ since $s^{a} s^{a}=0$ and $G^{a}(y) G^{b}(z) \rightarrow 2(y-z)^{-1} \delta^{a b} T(z)$ where

$$
T=\frac{1}{2} \Pi^{-}+v^{j} \Pi^{j}+\frac{1}{2} v^{j} v^{j} \Pi^{+}+t_{(0)}^{\dot{a}}\left(\gamma^{+} d\right)^{\dot{a}}+\sum_{n=0}^{\infty}\left(v_{(n+1)}^{j} w_{(n)}^{j}+t_{(n+1)}^{\dot{a}} u_{(n)}^{\dot{a}}\right) .
$$

One can treat $s^{a} s^{a}=0$ as a BRST constraint by defining $Q^{\prime}=\int d z\left[s^{a} G^{a}-b s^{a} s^{a}+c T\right]$ where $(b, c)$ are fermionic ghosts of dimension $(1,0)$. One can check that $Q^{\prime}$ is nilpotent with unconstrained $s^{a}$, and has cohomology equal to that of $Q$. Note that the algebra of $G^{a}$ and $T$ is not the usual $N=8$ superconformal algebra since, for example, $T$ has dimension 1 and commutes with $G^{a}$. Nevertheless, since $T$ and $G^{a}$ are first-class constraints, they can be used to gauge-fix $x^{+}=P^{+} \tau$ and $\left(\gamma^{+} \theta\right)^{a}=0$, and solve for $x^{-}$and $\left(\gamma^{-} p\right)^{a}$.

The only remaining constraint is that physical operators must commute with the zero mode of $T$, which in this light-cone gauge is $T_{0}+\frac{1}{2} P^{-}$where

$T_{0}=\int d z\left[-\frac{1}{2} \theta \gamma^{-} \partial \theta+v_{(0)}^{j} \partial x^{j}+\frac{1}{2} v_{(0)}^{j} v_{(0)}^{j} P^{+}+t_{(0)}^{\dot{a}}\left(\gamma^{+} d\right)^{\dot{a}}+\sum_{n=0}^{\infty}\left(v_{(n+1)}^{j} w_{(n)}^{j}+t_{(n+1)}^{\dot{a}} u_{(n)}^{\dot{a}}\right)\right]$

and a Lorentz frame has been chosen in which $P^{j}=0$ for $j=1$ to 8 . So physical operators are constructed from products of eigenvectors of $T_{0}$ whose $(\text { mass })^{2}=P^{+} P^{-}$is equal to the sum of the eigenvalues multiplied by $-2 P^{+}$. Since $T_{0}$ is quadratic, the bosonic 
and fermionic eigenvectors with eigenvalues $N, a_{N}$ and $b_{N}$, can be expressed as linear combinations of the remaining light-cone variables,

$$
\begin{gathered}
a_{N}=\int d \sigma\left[f_{N}^{j} \partial x^{j}+\sum_{n=0}^{\infty}\left(g_{N(n)}^{j} v_{(n)}^{j}+h_{N(n)}^{j} w_{(n)}^{j}\right)\right] \\
b_{N}=\int d \sigma\left[j_{N}^{\dot{a}}\left(\gamma^{+} p\right)^{\dot{a}}+k_{N}^{\dot{a}}\left(\gamma^{-} \theta\right)^{\dot{a}}+\sum_{n=0}^{\infty}\left(l_{N(n)}^{\dot{a}} \dot{t}_{(n)}^{\dot{a}}+m_{N(n)}^{\dot{a}} u_{(n)}^{\dot{a}}\right)\right] .
\end{gathered}
$$

If one requires that these eigenvectors satisfy the normalization condition that $\int d \sigma\left(f_{N}^{j} f_{N}^{j}+\right.$ $\left.\sum_{n=0}^{\infty} g_{N(n)}^{j} h_{N(n)}^{j}\right)$ and $\int d \sigma\left(j_{N}^{\dot{a}} k_{N}^{\dot{a}}+\sum_{n=0}^{\infty} l_{N(n)}^{\dot{a}} m_{N(n)}^{\dot{a}}\right)$ are finite, one finds that the only normalizable eigenvectors are the modes of

$$
y^{j}=\partial x^{j}+\sum_{n=0}^{\infty}\left(P^{+}\right)^{-n-1} \partial^{n+1} w_{(n)}^{j}, \quad q^{\dot{a}}=\left(\gamma^{+} p-\frac{1}{2} P^{+} \gamma^{-} \theta\right)^{\dot{a}}+\sum_{n=0}^{\infty}\left(P^{+}\right)^{-n-1} \partial^{n+1} u_{(n)}^{\dot{a}} .
$$

These eight bosonic and eight fermionic eigenvectors generate the usual light-cone GS spectrum. So the $T_{0}$ constraint has imposed the second-class constraints as well as the mass-shell condition. It is interesting to note that an infinite set of fields has also been useful for treating the second-class constraints of the chiral boson [17] and the self-dual Type IIB four-form [18].

\section{Superstring in $A d S_{5} \times S^{5}$ Background}

To construct the superstring action in a curved Type II supergravity background, one adds the integrated form of the closed massless vertex operator of section 4 to the superstring action in a flat background, and covariantizes with respect to superreparameterization invariance. As usual, one also needs to include a Fradkin-Tseytlin term for coupling the dilaton to the worldsheet curvature $r$. The resulting action is [7] [19]

$$
\begin{gathered}
S=\int d^{2} z\left[\frac{1}{2} \partial Y^{M} \bar{\partial} Y^{N}\left(G_{M N}(Y)+B_{M N}(Y)\right)\right. \\
\left.+\bar{\partial} Y^{M} \widetilde{d}_{\alpha} E_{M}^{\alpha}(Y)+\partial Y^{M} \widetilde{\widehat{d}} \widehat{\alpha} E_{M}^{\widehat{\alpha}}(Y)+\widetilde{d}_{\alpha} \widetilde{d_{\widehat{\beta}}} F^{\alpha \widehat{\beta}}(Y)+\alpha^{\prime} r \Phi(Y)\right]+S_{\lambda}+S_{\widehat{\lambda}}
\end{gathered}
$$

where $Y^{M}=\left(x^{m}, \theta^{\mu}, \widehat{\theta^{\mu}}\right)$ parameterizes the curved $\mathrm{N}=2$ superspace background, the first line of (7.1) is the usual GS action, $\widetilde{d}_{\alpha}=d_{\alpha}+N_{m n}\left(\gamma^{m n} D\right)_{\alpha}$ and $\widetilde{\widehat{d}}_{\widehat{\alpha}}=\widehat{d}_{\widehat{\alpha}}+\widehat{N}_{m n}\left(\gamma^{m n} \widehat{D}\right)_{\widehat{\alpha}}$ where $D_{\alpha}$ and $\widehat{D}_{\widehat{\alpha}}$ are $N=2$ superspace derivatives which act on the background fields to 
their right, $S_{\lambda}$ and $S_{\widehat{\lambda}}$ are the actions in a flat background for $\lambda^{\alpha}$ and $\widehat{\lambda}^{\widehat{\alpha}}$, and the lowest components of the superfields $E_{m}^{\alpha}$ and $E_{m}^{\widehat{\alpha}}$ are the gravitini, of $F^{\alpha \widehat{\beta}}$ are the RamondRamond field strengths, and of $\Phi$ is the dilaton. It is convenient to treat $d_{\alpha}$ and $\widehat{d}_{\widehat{\alpha}}$ (instead of $p_{\alpha}$ and $\widehat{p}_{\widehat{\alpha}}$ ) as fundamental worldsheet fields when the background is curved. Although the above action does not have $\kappa$-symmetry, it is constructed such that $\lambda^{\alpha} d_{\alpha}$ and $\widehat{\lambda}^{\alpha} \widehat{d}_{\widehat{\alpha}}$ are holomorphic and anti-holomorphic currents when the background superfields are on-shell. One can therefore quantize the action using $Q=\int d z \lambda^{\alpha} d_{\alpha}$ and $\widehat{Q}=\int d \bar{z} \widehat{\lambda^{\alpha}} \widehat{d} \widehat{\alpha}$ as in the flat case.

In the $A d S_{5} \times S^{5}$ background with $n$ units of Ramond-Ramond flux, $F^{\alpha \widehat{\beta}}=n \gamma_{01234}^{\alpha \widehat{\beta}}=$ $n \delta^{\alpha \widehat{\beta}}$, so the term $n \delta^{\alpha \widehat{\beta}} d_{\alpha} \widehat{d}_{\widehat{\beta}}$ in (7.1) allows one to solve the equations of motion [20] for $d_{\alpha}$ and $\widehat{d}_{\widehat{\alpha}}$ in terms of $\left(x^{m}, \theta^{\alpha}, \widehat{\theta}^{\alpha}\right)$. Plugging in the appropriate values for the $A d S_{5} \times S^{5}$ background superfields, one obtains [7][21] [22]

$$
S=\frac{1}{n^{2} g_{s}^{2}} \int d^{2} z\left[\frac{1}{2} \eta_{c d} J^{c} \bar{J}^{d}+\frac{1}{4} \delta_{\alpha \widehat{\beta}}\left(3 J^{\widehat{\beta}} \bar{J}^{\alpha}-J^{\alpha} \bar{J}^{\widehat{\beta}}\right)+N_{c d} J^{c d}+\widehat{N}_{c d} \bar{J}^{c d}\right]+S_{\lambda}+S_{\widehat{\lambda}}
$$

where $J^{A}=\left(g^{-1} \partial g\right)^{A}$ and $\bar{J}^{A}=\left(g^{-1} \bar{\partial} g\right)^{A}$ with $A=(a, \alpha, \widehat{\alpha}, c d)$ are left-invariant currents constructed from the coset supergroup $g(x, \theta, \widehat{\theta}) \in \frac{P S U(2,2 \mid 4)}{S O(4,1) \times S O(5)}$. This action is invariant under $g \rightarrow M g \Omega$ where $M$ is a global $P S U(2,2 \mid 4)$ transformation and $\Omega$ is a local $S O(4,1) \times$ $S O(5)$ transformation which also acts as a Lorentz rotation on the pure spinors $\lambda^{\alpha}$ and $\widehat{\lambda}^{\alpha}$. It has been checked to one-loop order [21] [14 that $S$ is conformally invariant and that

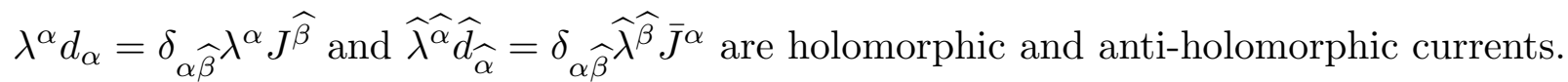

To construct the vertex operator $U=\lambda^{\alpha} \widehat{\lambda}^{\widehat{\beta}} A_{\alpha \widehat{\beta}}$ for fluctuations around the $A d S_{5} \times S^{5}$ background, one needs to generalize the equations of motion and gauge invariances of (4.5) as was done in 23 for the $A d S_{3} \times S^{3}$ background. The flat space equations of (4.5) can be generalized to $A d S_{5} \times S^{5}$ by simply replacing $D_{\alpha}$ and $\widehat{D}_{\widehat{\alpha}}$ in (4.5) with $\nabla_{\alpha}=$ $E_{\alpha}^{M}\left(\partial_{M}+\omega_{M}\right)$ and $\widehat{\nabla}_{\widehat{\alpha}}=E_{\widehat{\alpha}}^{M}\left(\partial_{M}+\omega_{M}\right)$ where $\omega_{M}^{c d}=\left(g^{-1} \partial_{M} g\right)^{c d}$ is the $S O(4,1) \times S O(5)$ spin connection and $E_{A}^{M}$ is the super-vierbein obtained by inverting $E_{M}^{A}=\left(g^{-1} \partial_{M} g\right)^{A}$ for $A=(a, \alpha, \widehat{\alpha})$. The equations of motion and gauge invariances are still self-consistent since, although $\left\{\nabla_{\alpha}, \widehat{\nabla}_{\widehat{\beta}}\right\}$ is non-zero in the $A d S_{5} \times S^{5}$ background, one can check that $\gamma_{m n p q r}^{\alpha \gamma}\left\{\nabla_{\alpha}, \widehat{\nabla}_{\widehat{\beta}}\right\} f_{\gamma}=0$ and $\gamma_{m n p q r}^{\widehat{\beta} \widehat{\gamma}}\left\{\nabla_{\alpha}, \widehat{\nabla}_{\widehat{\beta}}\right\} \widehat{f}_{\widehat{\gamma}}=0$ for any $f_{\gamma}$ and $\widehat{f}_{\widehat{\gamma}}$ and for any five-form direction mnpqr [10]. 


\section{Open Problems and Applications}

In this review, it was argued that pure spinors are the worldsheet ghosts of the GS formalism and that physical states are described by ghost-number one vertex operators in the cohomology of $Q=\int d z \lambda^{\alpha} d_{\alpha}$. Although these ideas were successfully used for computing N-point tree amplitudes, a more geometrical understanding would be useful for computing loop amplitudes where the ghosts play a more important role. In particular, one would like to understand how to formulate the action in a reparameterization-invariant manner and how the ghosts arise from the gauge-fixing procedure.

The relatively simple form of the vertex operator for fluctuations around the $A d S_{5} \times$ $S^{5}$ background suggests that it might be possible to compute tree amplitudes in this background. Although the currents $J^{A}$ are not holomorphic since $\bar{\partial} J^{A}=h_{B C}^{A} J^{B} \bar{J}^{C}$ where $h_{B C}^{A}$ are constants [24] [20], conformal invariance together with the $A d S$ isometries may be enough to imply their OPE's. Of course, even knowing these OPE's, one would still have to compute the zero mode contribution from $\left(x^{m}, \theta^{\mu}, \widehat{\theta}^{\mu}\right)$ to the tree amplitude, which might be complicated in an $A d S_{5} \times S^{5}$ background [25].

Acknowledgements: I would like to thank Michael Bershadsky, Osvaldo Chandía, Warren Siegel, Brenno Vallilo, and Edward Witten for useful conversations, and CNPq grant 300256/94-9 and the Clay Mathematics Institute for partial financial support. This research was partially conducted during the period the author was employed by the Clay Mathematics Institute as a CMI Prize Fellow. 


\section{References}

[1] M.B. Green and J.H. Schwarz, Supersymmetrical Dual String Theory, Nucl. Phys. B181 (1981) 502.

[2] D. Friedan, E. Martinec and S. Shenker, Conformal Invariance, Supersymmetry and String Theory, Nucl. Phys. B271 (1986) 93.

[3] M.B. Green and J.H. Schwarz, Covariant Description of Superstrings, Phys. Lett. B136 (1984) 367.

[4] N. Berkovits, Covariant Quantization Of The Green-Schwarz Superstring in a CalabiYau Background, Nucl. Phys. B431 (1994) 258, hep-th/9404162.

[5] N. Berkovits and C. Vafa, N=4 Topological Strings, Nucl. Phys. B433 (1995) 123, hep-th/9407190.

[6] W. Siegel, Classical Superstring Mechanics, Nucl. Phys. B263 (1986) 93.

[7] N. Berkovits, Super-Poincaré Covariant Quantization of the Superstring, JHEP 04 (2000) 018, hep-th/0001035.

[8] N. Berkovits and B.C. Vallilo, Consistency of Super-Poincaré Covariant Superstring Tree Amplitudes, JHEP 07 (2000) 015, hep-th/0004171.

[9] N. Berkovits, Cohomology in the Pure Spinor Formalism for the Superstring, hepth/0006003.

[10] N. Berkovits and Osvaldo Chandía, to appear.

[11] F. Essler, M. Hatsuda, T. Kimura, E. Laenen, A. Mikovic, W. Siegel and J. Yamron, Covariant Quantization of the First Ilk Superparticle, Nucl. Phys. B364 (1991) 67.

[12] P.S. Howe, Pure Spinor Lines in Superspace and Ten-Dimensional Supersymmetric Theories, Phys. Lett. B258 (1991) 141, Addendum-ibid.B259 (1991) 511;

P.S. Howe, Pure Spinors, Function Superspaces and Supergravity Theories in Ten Dimensions and Eleven Dimensions, Phys. Lett. B273 (1991) 90.

[13] B.E.W. Nilsson, "Pure Spinors as Auxiliary Fields in the Ten-Dimensional Supersymmetric Yang-Mills Theory", Class. Quant. Grav. 3 (1986) L41.

[14] M. Bershadsky, private communication.

[15] W. Siegel, Superfields in Higher-Dimensional Spacetime, Phys. Lett. 80B (1979) 220.

[16] N. Berkovits, M. Hatsuda and W. Siegel, The Big Picture, Nucl. Phys. B371 (1992) 434, hep-th/9108021.

[17] B. McClain, Y.S. Wu and F. Yu, Covariant Quantization of Chiral Bosons and OSp (1,1/2) Symmetry, Nucl. Phys. B343 (1990) 689;

C. Wotzasek, The Wess-Zumino Term for Chiral Bosons, Phys. Rev. Lett. 66 (1991) 129.

[18] N. Berkovits, Manifest Electromagnetic Duality in Closed Superstring Field Theory, Phys. Lett. B388 (1996) 743, hep-th/9607070. 
[19] N. Berkovits and W. Siegel, Superspace Effective Actions for $4 D$ Compactifications of Heterotic and Type II Superstrings, Nucl. Phys. B462 (1996) 213, hep-th/9510106.

[20] N. Berkovits, C. Vafa and E. Witten, Conformal Field Theory of AdS Background with Ramond-Ramond Flux, JHEP 9903 (1999) 018, hep-th/9902098.

[21] N. Berkovits, M. Bershadsky, T. Hauer, S. Zhukov and B. Zwiebach, Superstring Theory on $A d S_{2} \times S^{2}$ as a Coset Supermanifold, Nucl. Phys. B567 (2000) 61, hepth/9907200.

[22] R. Metsaev and A. Tseytlin, Type IIB superstring action in $A d S_{5} \times S^{5}$ background, Nucl.Phys. B533 (1998) 109, hep-th/9805028.

[23] L. Dolan and E. Witten, Vertex Operators for Ad $S_{3} \times S^{3}$ Background with RamondRamond Flux, JHEP 9911 (1999) 003, hep-th/9910205.

[24] M. Bershadsky, S. Zhukov and A. Zaintrob, PSL $(n \mid n)$ Sigma Model as a Conformal Field Theory, Nucl. Phys. B559 (1999) 205, hep-th/9902180.

[25] E. Witten, private communication. 